\title{
A simple risk score for mortality including the PCR Ct value upon admission in patients hospitalized due to COVID-19
}

\author{
Luis Kurzeder ${ }^{1} \cdot$ Rudolf A. Jörres $^{2} \cdot$ Thomas Unterweger $^{1}$. Julian Essmann ${ }^{1} \cdot$ Peter Alter $^{3} \cdot$ Kathrin Kahnert $^{4}$. \\ Andreas Bauer ${ }^{5} \cdot$ Sebastian Engelhardt ${ }^{1} \cdot$ Stephan Budweiser $^{1}[$
}

Received: 14 October 2021 / Accepted: 10 February 2022 / Published online: 26 February 2022

(c) The Author(s), under exclusive licence to Springer-Verlag GmbH Germany 2022

\begin{abstract}
Purpose To develop a simple score for the outcomes from COVID-19 that integrates information obtained at the time of admission including the $\mathrm{Ct}$ value (cycle threshold) for SARS-CoV-2.

Methods Patients with COVID-19 hospitalized from February 1st to May 31st 2021 in RoMed hospitals, Germany, were included. Clinical and laboratory parameters upon admission were recorded and patients followed until discharge or death. Logistic regression analysis was used to determine predictors of outcomes. Regression coefficients were used to develop a risk score for death.

Results Of 289 patients (46\% female, median age 66 years), 29\% underwent high-flow nasal oxygen (HFNO) therapy, 28\% were admitted to the Intensive Care Unit (ICU, $51 \%$ put on invasive ventilation, IV), and $15 \%$ died. Age $>70$ years, oxygen saturation $\leq 90 \%$, oxygen supply upon admission, eGFR $\leq 60 \mathrm{ml} / \mathrm{min}$ and $\mathrm{Ct}$ value $\leq 26$ were significant $(p<0.05$ each) predictors for death, to which 2, 2, 1, 1 and 2 score points, respectively, could be attributed. Sum scores of $\geq 4$ or $\geq 5$ points were associated with a sensitivity of $95.0 \%$ or $82.5 \%$, and a specificity of $72.5 \%$ or $81.7 \%$ regarding death. The high predictive value of the score was confirmed using data obtained between December 15th 2020 and January 31 st $2021(n=215)$.

Conclusion In COVID-19 patients, a simple scoring system based on data available shortly after hospital admission including the $\mathrm{Ct}$ value had a high predictive value for death. The score may also be useful to estimate the likelihood for required interventions at an early stage.
\end{abstract}

Keywords SARS-CoV-2 $\cdot$ COVID-19 $\cdot$ Risk score $\cdot$ Ct value $\cdot$ Mortality

Stephan Budweiser

Stephan.budweiser@ro-med.de

1 Department of Internal Medicine III, Division of Pulmonary and Respiratory Medicine, RoMed Hospital Rosenheim, Pettenkoferstrasse 10, 83022 Rosenheim, Germany

2 Institute and Outpatient Clinic for Occupational, Social and Environmental Medicine, Member of the German Center for Lung Research (DZL), University Hospital, LMU Munich, Comprehensive Pneumology Center Munich (CPC-M), Munich, Germany

3 Department of Medicine, Pulmonary and Critical Care Medicine, Member of the German Center for Lung Research (DZL), University of Marburg (UMR), Marburg, Germany

4 Department of Medicine V, Member of the German Center for Lung Research (DZL), University Hospital, LMU Munich, Comprehensive Pneumology Center Munich (CPC-M), Munich, Germany

5 Institute for Anesthesiology and Surgical Intensive Care Medicine, RoMed Hospital Rosenheim, Rosenheim, Germany

\section{Introduction}

A considerable number of patients infected with SARSCoV-2 develop severe symptoms leading to hospital admission, Intensive Care Unit (ICU) treatment and death from coronavirus disease 2019 (COVID-19) [1, 2]. Many studies have identified risk factors for a variety of outcomes. Older age, male sex and a higher burden from comorbidities [3-5], particularly chronic renal failure [6], turned out to be of major importance, in addition to generic factors such as treatment limitations [7], biomarkers, or radiological findings $[8,9]$ including their changes over time during hospital stay $[5,10-13]$. The respiratory viral load, as an indicator of the magnitude of the challenge by the virus, could also be a risk factor. This load is obtained by nasopharyngeal swabs and commonly quantified via the $\mathrm{Ct}$ (cycle threshold) value needed in the reverse transcription polymerase chain reaction (RT-PCR) of specific genes of SARS-CoV-2 to achieve 
a pre-determined signal strength [14]. The higher is the Ct value, the lower the virus load, and vice versa [15]. A number of studies found the $\mathrm{Ct}$ value to be predictive for mortality and ICU admission [16-18]. However, respiratory virus load is known to change during the course of infection, indicating the degree of virus shedding in an individual patient. Based on these limitations as well as methodological concerns [19], it has been suggested that the value in predicting the severity of COVID-19 is limited [20], while computed tomography and laboratory markers have been shown to be other important predictors [5, 21]. To avoid the difficulties arising from the time course of the $\mathrm{Ct}$ value, we focused on the value obtained as early as possible at hospital admission.

For the prediction of clinical outcomes of COVID-19, several models have been proposed [22-39]. As far as an initial tentative assessment is envisaged, scores should be simple and rely only on information available at the time of admission. At later stages during the hospital stay, information about changes in biomarkers or radiological findings might become more important [40-42]. To our knowledge, none of the proposed simple scoring systems for early assessment includes the $\mathrm{Ct}$ value obtained at the time of hospital admission, although it might be expected that its inclusion improves the accuracy of prediction. Thus, we analyzed data from patients hospitalized due to COVID-19, to develop a simple scoring system for mortality risk based on information available at the time of hospital admission including the $\mathrm{Ct}$ value. For comparison, we also determined the association of the predictors with other clinical outcomes, including the need for high-flow nasal oxygen (HFNO) therapy, requirement of ICU therapy, and invasive ventilation (IV).

\section{Methods}

\section{Study population}

Data were collected in the RoMed hospitals located in Rosenheim, Bad Aibling, Prien am Chiemsee and Wasserburg. Patients admitted to one of these hospitals between February 1st and May 31st 2021 (primary study population) and giving informed consent to the study protocol were enrolled. For validation, we used data from patients admitted between December 15th 2020 and January 31st 2021. The study protocol was approved by the Ethical Committee of the University Hospital of the LMU Munich (number 21-0452).

\section{Assessments}

Patients were characterized according to age, sex and vital parameters such as body temperature, heart rate, blood pressure, oxygen saturation from pulse oximetry $\left(\mathrm{SpO}_{2}\right)$ and respiratory rate upon admission. Comorbidities were recorded following the Charlson comorbidity index (CCI) without age, as age was used as a separate predictor. For patient characterization, we also used standard laboratory parameters, including leukocyte count, C-reactive protein (CRP), procalcitonin (PCT), hemoglobin, lactate dehydrogenase $(\mathrm{LDH})$, creatinine, troponin, $\mathrm{N}$-terminal pro b-type natriuretic peptide (NT-proBNP), D-dimer, alanine transaminase (ALT), aspartate aminotransferase (AST), gamma-glutamyltransferase (gamma-GT), lactate, in addition to the blood gas parameters of $\mathrm{pH}$, arterial pressures of oxygen $\left(\mathrm{pO}_{2}\right)$ and carbon dioxide $\left(\mathrm{pCO}_{2}\right)$, and standard base excess $(\mathrm{BE})$. The estimated glomerular filtration rate (eGFR) was determined from creatinine values according to the Chronic Kidney Disease-Epidemiology Collaboration (CKD-EPI) [43]. The presence of SARS-CoV-2 infection was determined via RTPCR from a nasopharyngeal swab taken at admission in the Clinical Laboratory of the Rosenheim Hospital, using the E gene and standardized procedures, and the number of cycles required to achieve a pre-determined threshold was quantified by the Ct value. Samples were taken into PCR buffer containing guanidine hydrochloride, using an aliquot for RNA isolation by means of the MagMAX ${ }^{\mathrm{TM}}$ Viral/Pathogen Nucleic Acid Isolation Kit (ThermoFisher, Life Technologies, Schwerte, Germany) and a KingFisher ${ }^{\mathrm{TM}}$ Flex Nukleic Acid Extraction System. The reverse transcription PCR (RTPCR) for the detection of SARS-CoV-2 RNA was performed via the ampliCube Coronavirus SARS-CoV-2 test (Mikrogen Diagnostik, Neuried, Germany). If sufficient material was available, the type of variant (wild type versus WHO alpha variant (B.1.1.7)) was determined by PCR kits for VirSNIP SARS-CoV-2 Spike del H69/V70 and VirSNIP SARSCoV-2 Spike N501Y (TIB MOLBIOL, Berlin, Germany).

We also recorded medical treatment including the potential administration of systemic steroids, anti-coagulation therapy and platelet aggregation inhibitors, including ICU admission, HFNO therapy and IV. All patients were followed until discharge from a RoMed hospital, discharge from another hospital after transfer (as far as compatible with data security requirements), or death.

\section{Outcomes}

The primary study outcome was death, additionally stratified for ICU and non-ICU stay. Secondary outcomes were the need for HFNO, ICU treatment and the clinical requirement of IV.

\section{Statistical analysis}

Numbers and percentages, or median values and quartiles were used for the description of the study population. To assess relationships with outcome measures, contingency tables and Chi-square statistics, as well as multiple binary 
logistic regression analysis were used. We aimed to define all predictors as binary variables to facilitate their translation into a score. The cutoff values of continuous variables were determined by ROC (receiver operator characteristics) analysis and the Youden criterion for each of the outcomes. All analyses were performed with the statistical software SPSS (Version 26, Armonk, NY, USA), and the level of statistical significance (two-sided) was assumed at $p<0.05$.

\section{Results}

\section{Study population}

The population was comprised of 289 patients $(46.0 \%$ female), with median [quartiles] age of 66.0 [51.0; 78.5] years (Table 1). Further data on laboratory parameters including $\mathrm{Ct}$ values are shown in the Supplemental Table S1, while data for medication and treatment modalities are given in Table 2. The median (quartiles) duration of hospitalization was $8.9(5.0 ; 14.9)$ days, and that of time to death 10.1

Table 1 Basic characteristics of patients

\begin{tabular}{lll}
\hline Demographic data & $n=289$ & $\begin{array}{l}\text { Percentage or } \\
\text { median [quartiles] }\end{array}$ \\
\hline Sex f/m & f: $133, \mathrm{~m}: 156$ & f: $46.0 \% \mathrm{m:} \mathrm{54.0 \%}$ \\
Age (years) & - & $66.0[51.0 ; 78.5]$ \\
Vital parameters & & \\
Heart rate (bpm) & - & $86[76 ; 99]$ \\
Systolic blood pressure $(\mathrm{mm} \mathrm{Hg})$ & - & $130[119 ; 145]$ \\
Diastolic blood pressure $(\mathrm{mm}$ & - & $80[70 ; 88]$ \\
Hg) & & \\
Body temperature $\left({ }^{\circ} \mathrm{C}\right)$ & - & $37.2[36.6 ; 38.9]$ \\
Oxygen saturation $\left(\mathrm{SpO}{ }_{2}\right)$ & - & $95[92 ; 97]$ \\
Presence of comorbidities & & \\
Arterial hypertension & 148 & $51.2 \%$ \\
Coronary artery disease & 26 & $9.0 \%$ \\
Left heart failure & 33 & $11.4 \%$ \\
Rhythm disorders & 39 & $13.5 \%$ \\
Diabetes mellitus & 55 & $19.0 \%$ \\
Renal failure & 33 & $11.4 \%$ \\
Cerebrovascular disease & 29 & $10.0 \%$ \\
Dementia & 17 & $5.9 \%$ \\
Mental disorder including & 38 & $13.1 \%$ \\
depression & & \\
Peripheral artery disease & 14 & $4.8 \%$ \\
Thrombosis/embolism & 16 & $5.5 \%$ \\
Any malignant disease & 16 & $5.5 \%$ \\
CCI w/o age & 289 & $1.0[0.0 ; 2.0]$ \\
CCI with age & 289 & $1.0 .0 ; 2.0]$ \\
\hline
\end{tabular}

CCI Charlson comorbidity score computed by either taking into account age (standard computation) or not. $f$ female, $m$ male
Table 2 Treatment of patients

\begin{tabular}{lll}
\hline & $n=289$ & Percentage \\
\hline Medication & & \\
Therapeutic: systemic steroids & 218 & $75.4 \%$ \\
Prophylactic: antiplatelet agents & 63 & $21.8 \%$ \\
Prophylactic: anti-coagulation therapy & 100 & $34.6 \%$ \\
Respiratory interventions & & \\
ICU patients (all) & 82 & $28.4 \%$ \\
High-flow nasal oxygen therapy (all) & 84 & $29.2 \%$ \\
$\quad$ Non-ICU* & 19 & $9.2 \%$ \\
$\quad$ ICU* & 65 & $80.2 \%$ \\
Invasive ventilation (ICU) & 42 & $51.2 \%$ \\
\hline
\end{tabular}

ICU Intensive Care Unit. Methods of ventilation were not mutually exclusive and several of them may have been consecutively applied in the same patient

*Subcategories of patients with high-flow nasal oxygen (HFNO) therapy

(5.0; 17.1) days. Regarding the variant of SARS-CoV-2, measurements were available in $73.4 \%$ of patients, showing that $7.3 \%$ of patients had the wild type and $66.1 \%$ the alpha variant.

Mean (95\% CI) Ct values for wild type, alpha variant and unknown were $26.8(23.9 ; 29.8), 26.1(25.2 ; 26.9)$ and 31.8 $(30.2 ; 33.4)$ cycles, respectively. The time course of variants is illustrated in the Supplemental Figure S1, demonstrating that the dominance of the alpha variant was already established during the initial phase of the time period analyzed.

\section{Analysis of risk factors}

We analyzed the data for the outcomes HFNO, ICU admission, IV and death (total, ICU, non-ICU), using only information easily available at the time of hospital admission or shortly afterwards. As such information we considered age, sex, $\mathrm{SpO}_{2}$, the presence of oxygen supply, renal function quantified via eGFR, and the $\mathrm{Ct}$ value (for other predictors see sensitivity analyses). Age, eGFR and $\mathrm{SpO}_{2}$ had already been established as risk factors in previous studies [7], while the importance of $\mathrm{Ct}$ values for mortality is illustrated in Fig. 1.

These predictors were used as binary scores for transformation into a scoring system, using optimal cutoff values determined by ROC analysis. Cutoff values of age $(>70$ years), $\mathrm{SpO}_{2}(\leq 90 \%)$ and eGFR $(\leq 60 \mathrm{~mL} / \mathrm{min})$ were the same for the outcomes death, HFNO, ICU admission and IV. Regarding the $\mathrm{Ct}$ value, the optimal cutoff value was $\leq 30$ for the outcomes HFNO and ICU treatment, and $\leq 26$ for IV and death (independent of ICU stay). The logistic regression analyses included these categorical variables, additionally the presence/absence of oxygen supply as predictors. Results are given in Table 3. 
Fig. 1 Ct values of PCR for SARS-CoV-2 plotted against age, with patients deceased from COVID-19 marked by black rhombi. It is evident that the $\mathrm{Ct}$ value was predictive for death especially in patients of age $>70$ years. $\mathrm{Ct}$ values were taken as provided by the laboratory irrespective of possibly exceeding extreme values of 40 . This was not relevant for the statistical analysis, since binary variables with cutoff values much below this extreme value were used

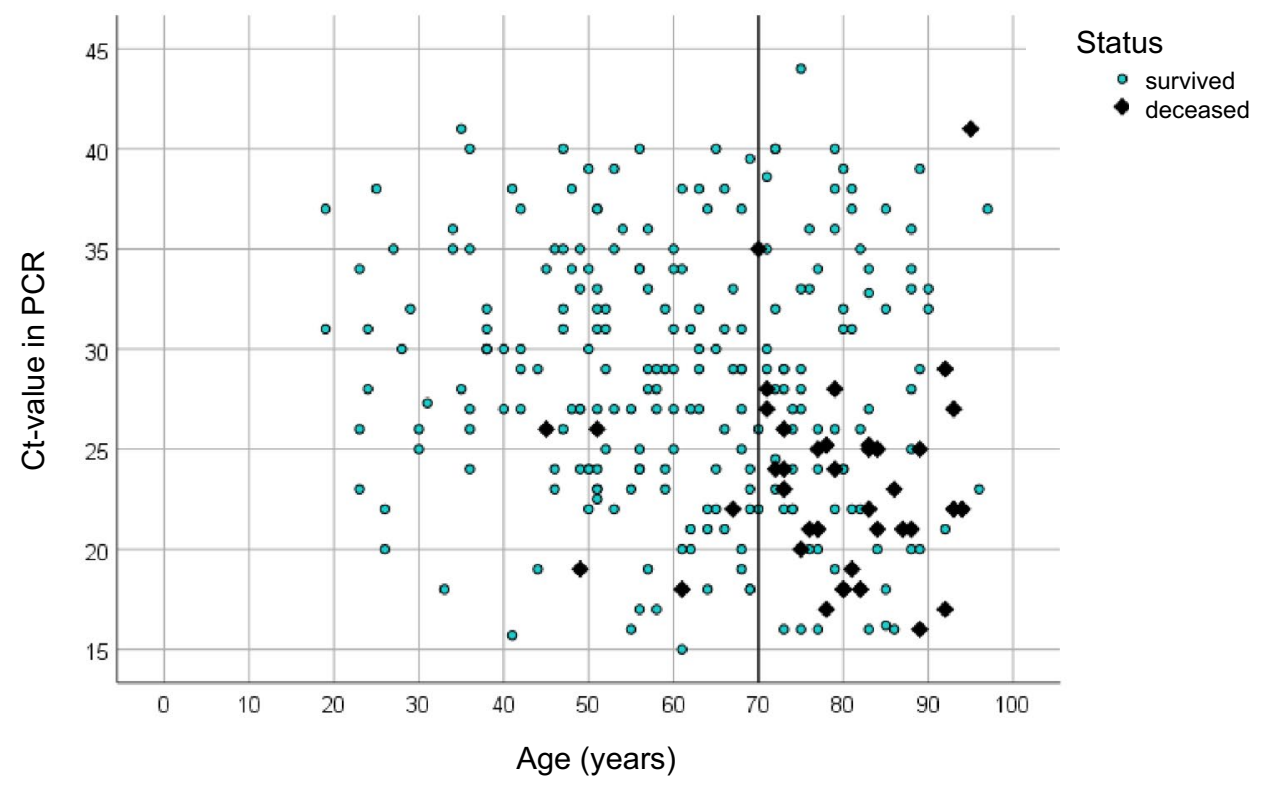

Table 3 Results of logistic regression analyses for the primary study population

\begin{tabular}{|c|c|c|c|c|c|c|}
\hline & $\begin{array}{l}\text { High-flow nasal } \\
\text { oxygen (HFNO) } \\
\text { therapy }\end{array}$ & $\begin{array}{l}\text { Admission to } \\
\text { Intensive Care } \\
\text { Unit (ICU) }\end{array}$ & $\begin{array}{l}\text { Intubation } \\
\text { required }\end{array}$ & $\begin{array}{l}\text { Number of } \\
\text { deceased } \\
\text { patients (all) }\end{array}$ & $\begin{array}{l}\text { Number of } \\
\text { deceased patients } \\
\text { on the ICU }\end{array}$ & $\begin{array}{l}\text { Number of deceased } \\
\text { patients outside the } \\
\text { ICU }\end{array}$ \\
\hline $\mathrm{n} /$ total $(\%)$ & $84 / 289(29.1 \%)$ & $82 / 289(28.4 \%)$ & $42 / 289(14.5 \%)$ & $44 / 289(15.2 \%)$ & $24 / 82(29.3 \%)$ & 20/207 (9.7\%) \\
\hline Age $(y)>70$ & 0.471 & $1.170 * *$ & 0.781 & $1.727 * *$ & $1.879 * *$ & 17.849 \\
\hline $\mathrm{SpO}_{2}(\%) \leq 90$ & $2.144 * * *$ & $2.575 * * *$ & $2.659 * * *$ & $2.028 * * *$ & $1.573^{*}$ & 1.114 \\
\hline $\begin{array}{l}\text { Requiring oxygen } \\
\text { on admission }\end{array}$ & $1.683 * * *$ & $1.810 * * *$ & $1.221 * *$ & $1.229 * *$ & 0.483 & 1.340 \\
\hline $\begin{array}{l}\mathrm{eGFR}(\mathrm{ml} / \\
\mathrm{min}) \leq 60\end{array}$ & 0.302 & 0.518 & 0.219 & 0.994* & 0.208 & $2.334 *$ \\
\hline $\mathrm{Ct} \leq 26$ & & & $1.021^{*}$ & $2.269 * * *$ & $1.856^{*}$ & $2.125^{*}$ \\
\hline $\mathrm{Ct} \leq 30$ & $1.278 * *$ & $1.471 * * *$ & & & & \\
\hline $\begin{array}{l}\text { Odds ratio for } \mathrm{Ct} \\
\text { from logistic } \\
\text { regression }\end{array}$ & $3.59 * *$ & $4.35 * * *$ & $2.78 *$ & $9.67 * * *$ & $6.40 *$ & $8.37 *$ \\
\hline \multicolumn{7}{|c|}{ Unadjusted odds ratios from contingency tables using the respective cutoff values for $\mathrm{Ct}$} \\
\hline $\begin{array}{l}\text { Odds ratio for } \mathrm{Ct} \\
\text { from contingency } \\
\text { table }\end{array}$ & $4.11 * * *$ & $4.40 * * *$ & $3.11^{*}$ & $7.72 * * *$ & $4.81 *$ & $8.92 * * *$ \\
\hline
\end{tabular}

The coefficients shown are the regression coefficients $(=\ln ($ Odds ratio)) which were used to define an additive risk score

${ }^{*} p<0.05, * * p<0.01, * * * p<0.001 . \mathrm{SpO}_{2}=$ oxygen saturation from pulse oximetry. Ctcycle threshold in PCR for SARS-CoV-2. All values refer to those obtained upon admission. In the lower part of the table for comparison, the unadjusted odds ratios derived from $2 \times 2$ contingency tables of each outcome versus the respective $\mathrm{Ct}$ value are shown, using the Chi-square statistics. The similarity of odds ratios obtained with the two approaches suggests little interference with the other determinants of the outcomes

The rounded integer values of the regression coefficients obtained for mortality (Table 3 ) were then used to define a novel additive risk score (Table 4). Using the coefficients for the number of all deceased patients, the following score points were chosen. We assigned 2 points for age $>70$ years, 2 points for $\mathrm{SpO}_{2} \leq 90 \%, 1$ point for oxygen supplementation, 1 point for eGFR $<60 \mathrm{~mL} / \mathrm{min}$, and 2 points for a $\mathrm{Ct}$ value $\leq 26$, each determined upon hospital admission. Table 4 also shows the numbers and percentages of patients, in whom the respective criterion was met, as well as sensitivity and specificity regarding death for each of the 5 items. For each patient, the score 
Table 4 Score points for each of the five categorical predictors of mortality showing the numbers and percentages of patients in whom the respective criterion was satisfied

\begin{tabular}{|c|c|c|c|c|c|c|c|c|c|}
\hline \multirow[t]{2}{*}{ Predictor } & \multirow[t]{2}{*}{ Score points $\$$} & \multicolumn{2}{|c|}{ All $(n=289)$} & \multicolumn{2}{|c|}{$\begin{array}{l}\text { Survivors } \\
(n=245)\end{array}$} & \multicolumn{2}{|c|}{ Deceased $(n=44)$} & \multirow[t]{2}{*}{ Sensitivity\& } & \multirow[t]{2}{*}{ Specificity ${ }^{\&}$} \\
\hline & & Number & $\% *$ & Number & $\% * *$ & Number & $\% * * *$ & & \\
\hline Age $(y)>70$ & 2 & 123 & 42.6 & 85 & 34.7 & 38 & 86.4 & 0.86 & 0.65 \\
\hline $\mathrm{SpO}_{2}(\%) \leq 90$ & 2 & 53 & 18.3 & 35 & 14.3 & 18 & 40.9 & 0.41 & 0.86 \\
\hline Oxygen required on admission & 1 & 97 & 33.6 & 73 & 29.8 & 24 & 54.5 & 0.55 & 0.70 \\
\hline eGFR $(\mathrm{ml} / \mathrm{min}) \leq 60$ & 1 & 100 & 34.6 & 67 & 27.3 & 33 & 75.0 & 0.75 & 0.73 \\
\hline $\mathrm{Ct}$ value $\leq 26$ & 2 & 129 & 44.6 & 94 & 38.4 & 35 & 79.5 & 0.80 & 0.62 \\
\hline
\end{tabular}

$\mathrm{SpO}_{2}$ oxygen saturation from pulse oximetry. $e G F R$ estimated glomerular filtration rate, $C t$ value cycle threshold value in PCR for SARS-CoV-2 E gene. *percentage of the total population showing the respective characteristic (e.g., age $>70$ years), **percentage of survivors showing the respective characteristic, *** percentage of deceased patients showing the respective characteristic. ${ }^{\&}$ Sensitivity and specificity for death as outcome, demonstrating that the 5 criteria had different contributions to the sum score regarding specificity and sensitivity. ${ }^{\$}$ Attributed on the basis of the (logarithmic) regression coefficients for death as outcome in all patients as shown in table

points could be summed up to obtain a risk score for mortality ranging from 0 to 8 .

The distribution in relation to mortality of this 5 -item score is depicted in Fig. 2, indicating a markedly increasing risk for death at score values $\geq 4$ or $\geq 5$. It was virtually absent for score values lower than 4 , but very high for values $\geq 6$. Sum scores of $\geq 4$ were associated with a sensitivity of $95.0 \%$ and a specificity of $72.5 \%$, and scores $\geq 5$ with values of $82.5 \%$ and $81.7 \%$ ( $p<0.001$ each). After computing a 4-item sum score without the $\mathrm{Ct}$ value, the distribution shown in the Supplemental Figure S2 was obtained, corresponding to a sensitivity and specificity of $90.2 \%$ and $68.2 \%$, respectively, for a value $\geq 3$, and of $68.3 \%$ and $87.7 \%$ for a value $\geq 4$. For the 5 -item score and cutoff values of $\geq 4$ or $\geq 5$, odds ratios for death for the 5-item score were 50 and 21 , respectively, whereby the latter number reflects that fact that the number of false-positive predictions increased with higher cutoff value (see Fig. 2). This comparison of the 5-item and the 4-item score was valid, as logistic regression analysis for death without the $\mathrm{Ct}$ value did not result in markedly altered coefficients for the other predictors, thus the same score points could be taken. The superiority in prediction was confirmed by logistic regression analysis including both sum scores as simultaneous predictors; the score including the $\mathrm{Ct}$ value was predictive $(p<0.0001)$ that without $\mathrm{Ct}$ value not $(p>0.5)$.

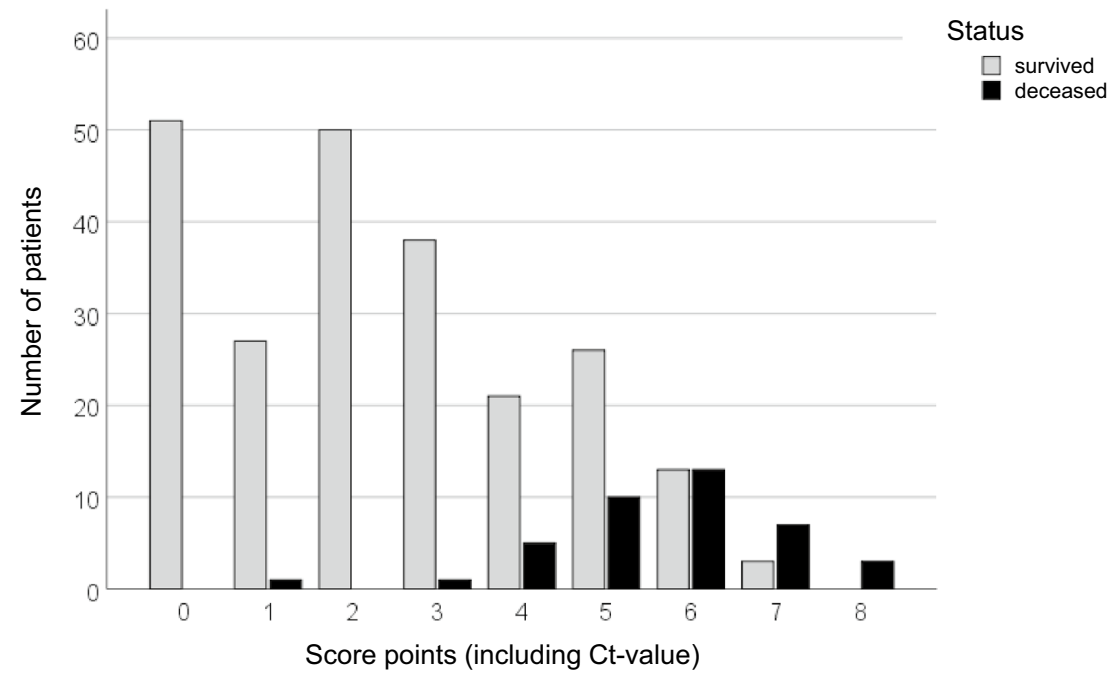

Fig. 2 Distribution of sum score values for mortality risk from five parameters available at the time of admission, including the $\mathrm{Ct}$ value (Table 3). It is evident that the cutoff value for prediction of mortality risk that yields high sensitivity and specificity can be chosen as 4 or 5 (for numeric values see "Results"). The patient with a score of 1 who died after 28 days of hospitalization with ICU admission, was a male ex-smoker of age 70 years with an BMI of $28 \mathrm{~kg} / \mathrm{m}^{2}$, the comorbidities diabetes and systemic hypertension, and a $\mathrm{Ct}$ value of 35 at the time of admission; the single score point originated from the need for supplemental oxygen at the time of admission 


\section{Sensitivity analyses and validation}

The CCI was omitted from the regression analysis as it would require a comprehensive assessment of clinical history which is often difficult upon admission. When performing additional analyses including the CCI, its predictive value was found to be absent or low (only relevant in case of HFNO). We also omitted sex as predictor since it turned out to be not relevant when added as a predictor to the five selected predictors. Furthermore, routine laboratory measures (Supplemental Table S1) were found not to be significant additional predictors when added as predictors to the five predictors given in Table 3, and the same was true for the blood gas parameters (Supplemental Table S1), as was true for vital signs such as heart rate and respiratory rate (Table 1). However, when analyzed separately without taking into account the five items of the score, the majority of laboratory parameters given in the Supplemental Table S1 differed between survivors and non-survivors (see legend to this table). Introducing the variant of SARS-CoV-2 (Supplemental Figure S1) as an additional categorical variable into the logistic regression analysis revealed that it was neither a significant predictor nor did it alter the significance level or the order of magnitude of the five basic predictors. The variant did, therefore, not interfere with the accuracy of prediction of death/survival.

To assess whether the score was critically dependent on the data used, we used data from patients admitted between December 15th 2020 and January 31st 2021. These patients showed characteristics very similar to those of the time period analyzed in the present study, and in addition it could be assumed that the pattern of virus variants was not much different from that observed in the primary study population (see Supplemental Figure S1). Data from 215 patients could be evaluated (47.9\% female, median age [quartiles] 76 [59, 84] years). The coefficients of logistic regression analysis were similar to those of Table 3, except for the fact that the coefficient referring to the use of oxygen supplementation upon admission was smaller and no longer statistically significant. When applying the score system derived from the primary study population to these data, the distribution shown in the Supplemental Figure S3 was obtained. It can be seen that the score was again predictive in these patients. A score $\geq 4$ was associated with a sensitivity of $85.7 \%$ and a specificity of $57.2 \%$, and a score $\geq 5$ with values of $71.4 \%$ and $70.5 \%$, respectively.

\section{Discussion}

We analyzed data from patients hospitalized between February 1st and May 31st 2021 due to COVID-19 in southern Germany, aiming to define a simple risk score that could be calculated from basic parameters available at hospital admission. The primary outcome studied was death. It turned out that the PCR Ct value for the presence of SARS-CoV-2, obtained from nasopharyngeal swabs, was an integral element of this score, in addition to age above 70 years, $\mathrm{SpO}_{2}$ of $90 \%$ or lower, the presence of oxygen supplementation, and impaired renal function as indicated by eGFR $\leq 60 \mathrm{ml} /$ min upon hospital admission. The relevant $\mathrm{Ct}$ value was 26 or less, indicating a high respiratory viral load. The proposed score for mortality risk (range $0-8$ ) is simple to compute and yielded high predictive accuracy when using cutoff values of 4 or 5. Regarding the additional outcomes HFNO and admission to ICU, a Ct value of 30 or less became predictive.

Several risk scores for mortality from COVID-19 or the need for treatment modalities have been proposed so far [23-39], but none of them explicitly included the Ct value as a measure of respiratory viral load. The outcomes that have been analyzed ranged from ICU admission and respiratory failure to mortality. In accordance with our findings, age was identified as a significant predictor across studies [23-28, $30-37,39]$. If its influence was quantified via cutoff values, these mostly ranged between 60 and 75 years $[25,26,30-32$, $36,37]$ and thus were not markedly different from the value of 70 years which we found adequate for all outcomes. Studies also included $\mathrm{SpO}_{2}$ as a predictor [24, 27, 30, 37, 39]. The present cutoff value of $90 \%$ upon admission was compatible with reported values under $94 \%$ [24, 26, 33, 37]. A number of patients already required supplementary oxygen before admission, thus ambient-air saturation values were not available. We found that the presence of oxygen supplementation upon admission could be easily incorporated as a predictor. While a saturation of $>90 \%$ was not counted in the score if achieved under supplementary oxygen, the fact that supplementary oxygen was administered was counted. Conversely, if $\mathrm{SpO} 2$ was $\leq 90 \%$ even with supplementary oxygen, the presence of supply increased the risk score. The two criteria were additive in the logistic regression analysis, without interaction between them, thus enabling them to be incorporated as additive contributions to the sum score.

In previous studies, elevated values of creatinine have been identified as relevant $[24,25,27,30]$, in accordance with our findings regarding eGFR. We favored the use of eGFR over creatinine as it includes a standardization for age and sex. In most previous analyses, the dependence of risk on creatinine was determined without giving explicit cutoff values or score points. The cutoff value of $\leq 60 \mathrm{ml} /$ min that we identified as optimal is in line with clinical recommendations [43], moreover similar to cutoff values that can be derived from a proposed cutoff value of $1.2 \mathrm{mg} / \mathrm{dl}$ for creatinine [27], assuming men with age 65-70 years.

Regarding Ct values, we found different cutoff values for the prediction of HFNO and ICU admission compared to IV and death. The observation that the optimal cutoff for HFNO 
and ICU admission was 30 seemed to reflect the fact that values above 30 can be considered as indicating a low risk. Conversely, we found $\mathrm{Ct}$ values of 26 or below, reflecting a high virus load, to be most informative for IV and especially death from COVID-19. This is illustrated in Fig. 1 indicating that mortality risk was very low in patients with high Ct values. In addition, the prediction of death/survival was more precise when including the $\mathrm{Ct}$ value (Fig. 2 vs. Supplemental Fig. 2), indicating that in the range of high sum scores, prediction was better when including the $\mathrm{Ct}$ value. When comparing the odds ratios from adjusted logistic regression analysis with those from unadjusted contingency tables (Table 3), their similarity suggested little interference of $\mathrm{Ct}$ values with the other determinants of the outcomes, thereby supporting their additive contribution. This was favored by the use of the logarithmic coefficients for the scoring system, as these are naturally additive, in contrast to odds ratios which are multiplicative.

Different findings about the predictive value of the $\mathrm{Ct}$ value have been reported. There are systematic reviews [16, 18 ] indicating a predictive role for morbidity and mortality, but studies have also pointed to a number of weaknesses, such as inter-individual differences in sample-taking, which may result in varying outcomes for specimens [19]. Moreover, Ct values change over the course of the disease, which renders them dependent on the time of sampling [23, 44]. We only took values assessed at an early stage upon hospital admission, and the result supports the assumption that initial values were already sufficient for a satisfactory prediction of outcomes, especially death. Despite this, consecutive PCR tests could further improve the predictive power.

When using data from a 1.5-month time period immediately prior to that covered by the present study to assess the validity of the proposed score, its predictive value turned out still to be high, thereby indicating that it was robust and applicable beyond the cohort from which it was derived. The score proposed did not include standard laboratory parameters or blood gases assessed at admission. When these were added as predictors to the regression analyses, they were not significant and the prediction was not improved. If analyzed separately without taking into account other parameters or any adjustment, most of them (see Supplemental Table S1) were associated with the outcome death. As a consequence, our findings are fully compatible with those of other studies in which parameters such as CRP, LDH and D-dimer were found to be linked to COVID-19 outcomes; they only underline the comprehensive nature of the five items comprised in the clinical score proposed by us. We also found the virus variant to be not relevant for the score regarding any of the outcomes analyzed, however, this analysis was limited by the fact that the number of patients infected with wild-type SARS-CoV-2 decreased considerably during the study period.

\section{Limitations}

The present analysis was based on data of COVID-19 patients admitted between February 1st and May 31st 2021 in southern Germany, a period which was dominated by the alpha variant of SARS-CoV-2, and it is not clear to which extent the score can be extrapolated to other regions and virus variants. The analysis of the second sample in principle confirmed the predictive value of the score and the rank order of predictors but was limited by the small sample size that was a consequence of the time restriction required to establish comparability between samples. Occurrence of novel variants over time is to be expected and might require adjustment of the Ct cutoff value and/ or the score points attributed to the PCR results. The fact, however, that the PCR information was additive relative to the information provided by the other four predictors, renders it likely that such adjustments, if needed, can easily be performed without disturbance of the scoring system. One might argue that the chosen secondary outcomes depend on clinical decisions and are not necessarily based on the same criteria in different locations. As we found secondary outcomes to be dependent on the same set of predictors as mortality risk, although with different coefficients, the present score might also be helpful to estimate the need for resource allocation upon hospital admission.

\section{Conclusion}

Using data from patients hospitalized due to COVID-19 between February 1st and May 31st 2021, we defined a simple mortality risk score from basic information available at the time of hospital admission. The proposed sum score for mortality risk ranges from 0 to 8 and is based on five simple predictors. Age $>70$ years, $\mathrm{SpO}_{2} \leq 90 \%$, the need for supplemental oxygen, and an eGFR $\leq 60 \mathrm{ml} /$ min were significant predictors; prediction was improved when additionally taking a $\mathrm{Ct}$ value $\leq 26$ for SARS-CoV-2 into account. The five items corresponded to 2, 2, 1, 1, and 2 score points, respectively. Using threshold values of 4 or 5 for the sum score, sensitivity/specificity was $95.0 \% / 72.5 \%$ and $82.5 \% / 81.7 \%$, respectively, with odds ratios for death $>20$. The scoring system provides a quick and simple tool that may be used for initial risk assessment, prior to the availability of further data obtained during the hospital stay.

Supplementary Information The online version contains supplementary material available at https://doi.org/10.1007/s15010-022-01783-1. 


\section{Declarations}

Conflict of interests The authors have no relevant financial or nonfinancial interests to disclose.

Ethics approval Approval was obtained from the ethics committee of the University Hospital of the LMU Munich (number 21-0452). The procedures used in this study adhered to the tenets of the Declaration of Helsinki.

Consent to participate Informed consent was obtained from all individual participants in the study or their legal guardians.

\section{References}

1. Li J, He X, Yuan Y, Zhang W, Li X, Zhang Y, et al. Meta-analysis investigating the relationship between clinical features, outcomes, and severity of severe acute respiratory syndrome coronavirus 2 (SARS-CoV-2) pneumonia. Am J Infect Control. 2021;49:82-9. https://doi.org/10.1016/j.ajic.2020.06.008.

2. Zhang JJY, Lee KS, Ang LW, Leo YS, Young BE. Risk factors for severe disease and efficacy of treatment in patients infected with COVID-19: a systematic review, meta-analysis, and metaregression analysis. Clin Infect Dis. 2020;71:2199-206. https:// doi.org/10.1093/cid/ciaa576.

3. Pijls BG, Jolani S, Atherley A, Derckx RT, Dijkstra JIR, Franssen GHL, et al. Demographic risk factors for COVID-19 infection, severity, ICU admission and death: a meta-analysis of 59 studies. BMJ Open. 2021;11: e044640. https://doi.org/10.1136/bmjop en-2020-044640.

4. Ng WH, Tipih T, Makoah NA, Vermeulen J-G, Goedhals D, Sempa JB, et al. Comorbidities in SARS-CoV-2 Patients: a systematic review and meta-analysis. MBio. 2021. https://doi.org/10. 1128/mBio.03647-20.

5. Xie J, Wang Q, Xu Y, Zhang T, Chen L, Zuo X, et al. Clinical characteristics, laboratory abnormalities and CT findings of COVID-19 patients and risk factors of severe disease: a systematic review and meta-analysis. Ann Palliat Med. 2021;10:1928-49. https://doi.org/10.21037/apm-20-1863.

6. Li Y, Ashcroft T, Chung A, Dighero I, Dozier M, Horne M, et al. Risk factors for poor outcomes in hospitalised COVID-19 patients: A systematic review and meta-analysis. J Glob Health. 2021;11:10001. https://doi.org/10.7189/jogh.11.10001.

7. Budweiser S, Baş Ș, Jörres RA, Engelhardt S, von Delius S, Lenherr K, et al. Patients' treatment limitations as predictive factor for mortality in COVID-19: results from hospitalized patients of a hotspot region for SARS-CoV-2 infections. Respir Res. 2021;22:168. https://doi.org/10.1186/s12931-021-01756-2.

8. Knight SR, Ho A, Pius R, Buchan I, Carson G, Drake TM, et al. Risk stratification of patients admitted to hospital with covid-19 using the ISARIC WHO Clinical Characterisation Protocol: development and validation of the 4C Mortality Score. BMJ. 2020;370: m3339. https://doi.org/10.1136/bmj.m3339.

9. Myrstad M, Ihle-Hansen H, Tveita AA, Andersen EL, Nygård S, Tveit A, Berge T. National Early Warning Score 2 (NEWS2) on admission predicts severe disease and in-hospital mortality from Covid-19 - a prospective cohort study. Scand J Trauma Resusc Emerg Med. 2020;28:66. https://doi.org/10.1186/ s13049-020-00764-3.

10. Shi C, Wang L, Ye J, Gu Z, Wang S, Xia J, et al. Predictors of mortality in patients with coronavirus disease 2019: a systematic review and meta-analysis. BMC Infect Dis. 2021;21:663. https:// doi.org/10.1186/s12879-021-06369-0.
11. Hashemi-Madani N, Emami Z, Janani L, Khamseh ME. Typical chest CT features can determine the severity of COVID-19: A systematic review and meta-analysis of the observational studies. Clin Imaging. 2021;74:67-75. https://doi.org/10.1016/j. clinimag.2020.12.037.

12. Zheng Y, Wang L, Ben S. Meta-analysis of chest CT features of patients with COVID-19 pneumonia. J Med Virol. 2021;93:241-9. https://doi.org/10.1002/jmv.26218.

13. Zhang L, Hou J, Ma F-Z, Li J, Xue S, Xu Z-G. The common risk factors for progression and mortality in COVID-19 patients: a meta-analysis. Arch Virol. 2021;166:2071-87. https://doi.org/ 10.1007/s00705-021-05012-2.

14. Colton H, Ankcorn M, Yavuz M, Tovey L, Cope A, Raza M, et al. Improved sensitivity using a dual target, E and RdRp assay for the diagnosis of SARS-CoV-2 infection: Experience at a large NHS Foundation Trust in the UK. J Infect. 2021;82:15998. https://doi.org/10.1016/j.jinf.2020.05.061.

15. Zou L, Ruan F, Huang M, Liang L, Huang H, Hong Z, et al. SARS-CoV-2 viral load in upper respiratory specimens of infected patients. N Engl J Med. 2020;382:1177-9. https://doi. org/10.1056/NEJMc2001737.

16. Shenoy S. SARS-CoV-2 (COVID-19), viral load and clinical outcomes; lessons learned one year into the pandemic: A systematic review. World J Crit Care Med. 2021;10:132-50. https:// doi.org/10.5492/wjccm.v10.i4.132.

17. Tanner AR, Phan H, Brendish NJ, Borca F, Beard KR, Poole S, W Clark T. SARS-CoV-2 viral load at presentation to hospital is independently associated with the risk of death. J Infect 2021. https://doi.org/10.1016/j.jinf.2021.08.003.

18. Rao SN, Manissero D, Steele VR, Pareja J. A systematic review of the clinical utility of cycle threshold values in the context of COVID-19. Infect Dis Ther. 2020;9:573-86. https://doi.org/10. 1007/s40121-020-00324-3.

19. Dahdouh E, Lázaro-Perona F, Romero-Gómez MP, Mingorance J, García-Rodriguez J. Ct values from SARS-CoV-2 diagnostic PCR assays should not be used as direct estimates of viral load. J Infect. 2021;82:414-51. https://doi.org/10.1016/j.jinf.2020. 10.017.

20. Biguenet A, Bouiller K, Marty-Quinternet S, Brunel A-S, Chirouze C, Lepiller Q. SARS-CoV-2 respiratory viral loads and association with clinical and biological features. J Med Virol. 2021;93:1761-5. https://doi.org/10.1002/jmv.26489.

21. Alsharif W, Qurashi A. Effectiveness of COVID-19 diagnosis and management tools: a review. Radiography (Lond). 2021;27:682-7. https://doi.org/10.1016/j.radi.2020.09.010.

22. Miki S, Sasaki H, Horiuchi H, Miyata N, Yoshimura Y, Miyazaki K, et al. On-admission SARS-CoV-2 RNAemia as a single potent predictive marker of critical condition development and mortality in COVID-19. PLoS ONE. 2021;16: e0254640. https://doi.org/10.1371/journal.pone.0254640.

23. Ageno W, Cogliati C, Perego M, Girelli D, Crisafulli E, Pizzolo $\mathrm{F}$, et al. Clinical risk scores for the early prediction of severe outcomes in patients hospitalized for COVID-19. Intern Emerg Med. 2021;16:989-96. https://doi.org/10.1007/ s11739-020-02617-4.

24. Alkaabi S, Alnuaimi A, Alharbi M, Amari MA, Ganapathy R, Iqbal I, et al. A clinical risk score to predict in-hospital mortality in critically ill patients with COVID-19: a retrospective cohort study. BMJ Open. 2021;11: e048770. https://doi.org/10.1136/ bmjopen-2021-048770.

25. Bartoletti M, Giannella M, Scudeller L, Tedeschi S, Rinaldi M, Bussini L, et al. Development and validation of a prediction model for severe respiratory failure in hospitalized patients with SARSCoV-2 infection: a multicentre cohort study (PREDI-CO study). Clin Microbiol Infect. 2020;26:1545-53. https://doi.org/10.1016/j. cmi.2020.08.003. 
26. Bennouar S, Bachir Cherif A, Kessira A, Bennouar D-E, Abdi $\mathrm{S}$. Development and validation of a laboratory risk score for the early prediction of COVID-19 severity and in-hospital mortality. Intensive Crit Care Nurs. 2021;64: 103012. https://doi.org/10. 1016/j.iccn.2021.103012.

27. Bertsimas D, Lukin G, Mingardi L, Nohadani O, Orfanoudaki A, Stellato B, et al. COVID-19 mortality risk assessment: An international multi-center study. PLoS ONE. 2020;15: e0243262. https://doi.org/10.1371/journal.pone.0243262.

28. Fumagalli C, Ungar A, Rozzini R, Vannini M, Coccia F, Cesaroni $\mathrm{G}$, et al. Predicting mortality risk in older hospitalized persons with COVID-19: a comparison of the COVID-19 mortality risk score with frailty and disability. J Am Med Dir Assoc. 2021;22:1588-1592.e1. https://doi.org/10.1016/j.jamda.2021.05. 028 .

29. Galloway JB, Norton S, Barker RD, Brookes A, Carey I, Clarke $\mathrm{BD}$, et al. A clinical risk score to identify patients with COVID-19 at high risk of critical care admission or death: An observational cohort study. J Infect. 2020;81:282-8. https://doi.org/10.1016/j. jinf.2020.05.064.

30. Gude F, Riveiro V, Rodríguez-Núñez N, Ricoy J, Lado-Baleato Ó, Lourido T, et al. Development and validation of a clinical score to estimate progression to severe or critical state in COVID-19 pneumonia hospitalized patients. Sci Rep. 2020;10:19794. https:// doi.org/10.1038/s41598-020-75651-z.

31. Gue YX, Tennyson M, Gao J, Ren S, Kanji R, Gorog DA. Development of a novel risk score to predict mortality in patients admitted to hospital with COVID-19. Sci Rep. 2020;10:21379. https:// doi.org/10.1038/s41598-020-78505-w.

32. Her AY, Bhak Y, Jun EJ, Yuan SL, Garg S, Lee S, et al. A clinical risk score to predict in-hospital mortality from COVID-19 in South Korea. J Korean Med Sci. 2021;36: e108. https://doi.org/ 10.3346/jkms.2021.36.e108.

33. Hu C, Liu Z, Jiang Y, Shi O, Zhang X, Xu K, et al. Early prediction of mortality risk among patients with severe COVID19, using machine learning. Int J Epidemiol. 2021;49:1918-29. https://doi.org/10.1093/ije/dyaa171.

34. Huang D, Wang T, Chen Z, Yang H, Yao R, Liang Z. A novel risk score to predict diagnosis with coronavirus disease 2019 (COVID-19) in suspected patients: A retrospective, multicenter, and observational study. J Med Virol. 2020;92:2709-17. https:// doi.org/10.1002/jmv.26143.

35. Jiang M, Li C, Zheng L, Lv W, He Z, Cui X, Dietrich CF. A biomarker-based age, biomarkers, clinical history, sex
(ABCS)-mortality risk score for patients with coronavirus disease 2019. Ann Transl Med. 2021;9:230. https://doi.org/10.21037/ atm-20-6205.

36. Kljakovic Gaspic T, Pavicic Ivelja M, Kumric M, Matetic A, Delic N, Vrkic I, Bozic J. In-hospital mortality of COVID-19 patients treated with high-flow nasal oxygen: evaluation of biomarkers and development of the novel risk score model CROW-65. Life (Basel). 2021. https://doi.org/10.3390/life11080735.

37. Levine DM, Lipsitz SR, Co Z, Song W, Dykes PC, Samal L. Derivation of a clinical risk score to predict 14-day occurrence of hypoxia, ICU admission, and death among patients with coronavirus disease 2019. J Gen Intern Med. 2021;36:730-7. https:// doi.org/10.1007/s11606-020-06353-5.

38. Liang W, Liang H, Ou L, Chen B, Chen A, Li C, et al. Development and validation of a clinical risk score to predict the occurrence of critical illness in hospitalized patients with COVID-19. JAMA Intern Med. 2020;180:1081-9. https://doi.org/10.1001/ jamainternmed.2020.2033.

39. López-Escobar A, Madurga R, Castellano JM, Velázquez S, Del Suárez VR, Menéndez J, et al. Risk score for predicting in-hospital mortality in COVID-19 (RIM Score). Diagnostics (Basel). 2021. https://doi.org/10.3390/diagnostics11040596.

40. Ponti G, Maccaferri M, Ruini C, Tomasi A, Ozben T. Biomarkers associated with COVID-19 disease progression. Crit Rev Clin Lab Sci. 2020;57:389-99. https://doi.org/10.1080/10408363.2020. 1770685.

41. Velavan TP, Kuk S, Le Linh TK, Lamsfus Calle C, Lalremruata A, Pallerla SR, et al. Longitudinal monitoring of laboratory markers characterizes hospitalized and ambulatory COVID19 patients. Sci Rep. 2021;11:14471. https://doi.org/10.1038/ s41598-021-93950-x.

42. Gallo Marin B, Aghagoli G, Lavine K, Yang L, Siff EJ, Chiang SS, et al. Predictors of COVID-19 severity: A literature review. Rev Med Virol. 2021;31:1-10. https://doi.org/10.1002/rmv.2146.

43. Levey AS, Stevens LA, Schmid CH, Zhang YL, Castro AF, Feldman HI, et al. A new equation to estimate glomerular filtration rate. Ann Intern Med. 2009;150:604-12. https://doi.org/10.7326/ 0003-4819-150-9-200905050-00006.

44. Singanayagam A, Patel M, Charlett A, Lopez Bernal J, Saliba V, Ellis J, et al. Duration of infectiousness and correlation with RT-PCR cycle threshold values in cases of COVID-19, England, January to May 2020. Euro Surveill. 2020. https://doi.org/10. 2807/1560-7917.ES.2020.25.32.2001483. 